\title{
EFEITOS DE DIFERENTES INTERVENÇÕES NO PROCESSO DE EUTROFIZAÇÃO DO LAGO PARANOÁ (BRASÍLIA - DF)
}

\author{
Ronaldo Angelini $1^{*}$, Luis Maurício Bini ${ }^{2}$ \& Fernando L.R.M. Starling ${ }^{3}$ \\ ${ }^{1}$ Universidade Estadual de Goiás, LAB - Laboratório de Pesquisa Ecológica e Educação Científica, BR 153 Km 98, CP 459, CEP 75074-840. Anápolis, \\ Goiás, Brasil. \\ ${ }^{2}$ Universidade Federal de Goiás, Departamento de Biologia Geral, CP 131, CEP 74001-970. Goiânia, Goiás, Brasil \\ ${ }^{3}$ Companhia de Abastecimento e Saneamento de Brasília (CAESB), Laboratório Central, Sain, Área Especial, CEP 70620-000. Brasília, Distrito \\ Federal, Brasil. \\ *E-mail: ronaldo.angelini@pq.cnpq.br
}

\section{RESUMO}

O monitoramento temporal das variáveis de um corpo d'água possibilita o conhecimento de sua dinâmica e, por fim, de seu manejo. O Lago Paranoá em Brasília, formado em 1959, passou por um longo período de eutrofização. Neste trabalho, usando dados de monitoramento de longa duração da CAESB, os efeitos da instalação de duas Estações de Tratamento de Esgoto (ETE) e de um evento de flushing foram avaliados para as variáveis Clorofila-a, Fósforo Total e Transparência medidas entre 1976 e 2001. Utilizando-se da Análise de Intervenção foram mensuradas a efetividade de três intervenções: o início das operações das ETE (01/1993); o pleno funcionamento destas ETE (01/1996); e um evento de flushing (abertura abrupta das comportas, 11/1999). Os resultados mostram que: i) a $1^{\mathrm{a}}$ intervenção não surtiu nenhum efeito; ii) a $2^{\mathrm{a}}$ intervenção fez com que os níveis de Fósforo Total e Clorofila-a fossem reduzidos significativamente; iii) apenas o flushing ( $3^{\mathrm{a}}$ intervenção), fez com que a transparência da água aumentasse para mais de um metro na maioria dos pontos, inferindo-se que apesar da redução do aporte de fósforo ao lago com as ETE ( $1^{\mathrm{a}}$ e $2^{\mathrm{a}}$ intervenções), a abundância restante de fitoplâncton na água tenha mantido um mecanismo de feedback com o fósforo que só foi interrompido com a abertura abrupta das comportas que eliminou o fitoplâncton da superfície e conduziu o lago à um novo patamar de produção primária. O programa de monitoramento da CAESB foi fundamental para o entendimento deste processo e o que justifica a manutenção e a ampliação deste programa.

Palavras-chave: intervenção, reservatório tropical, CAESB, monitoramento, Paranoá.

\begin{abstract}
EFFECTS OF INTERVENTIONS ON THE EUTROPHICATION PROCESS OF PARANOÁ LAKE RESERVOIR OF BRASILIA, BRAZIL. Long-term environmental monitoring of aquatic ecosystems is essential to understand their dynamics and to the proposition of management practices. Paranoá Reservoir was created in 1959 and was subject to a long period of eutrophication. In this paper, data obtained by the monitoring program carried out by the "Companhia de Abastecimento e Saneamento de Brasília", between 1976 and 2001, were evaluated to measure the effects of three direct interventions on water quality (as measured by water transparency, P-total and chlorophyll-a concentrations): the activation of two water treatment plants (01/1993); the proper operation of these plants (01/1996), and a major flushing event (sudden release of water in 11/1999). The following results were obtained: i) the first intervention had no effect on the mean values of the variables analyzed; ii) the chlorophyll-a and total phosphorus concentrations significantly declined after the second intervention; iii) water transparency increased after the third intervention. An apparent mechanism of phosphorus feedback supported by phytoplankton was interrupted by the flushing and, after that intervention, the primary production of Paranoá Reservoir declined to a new level. The dataset obtained by the monitoring program was essential to understand this process and, therefore, the continuity (and even amplification) of this program was fully justified.
\end{abstract}

Keywords: environmental monitoring, intervention analysis, primary production, tropical water reservoir. 


\section{INTRODUÇÃO}

O monitoramento ambiental pode ser definido como uma série temporal de medições de variáveis físicas, químicas e biológicas escolhidas para responder questões sobre mudanças no ecossistema (Lovett et al. 2007). Mais especificamente biomonitoramento é a medição do comportamento de atributos de organismos vivos a fim de avaliar as alterações ocorridas no ambiente (Matthews et al. 1982).

Dentre os exemplos mais conhecidos de biomonitoramento, estão: a diminuição da diversidade de grupos que servem como indicadores biológicos (Arias et al. 2007), as alterações na abundância de espécies-alvo da atividade pesqueira (Milessi et al. 2005) ou ainda a análise de eventos de eutrofização de corpos d'água que, em geral, sugerem alta influência antropogênica na bacia hidrográfica (Shigaki et al. 2006).

No que diz respeito à este último tema, sua importância é crucial para a avaliação do desenvolvimento de ecossistemas aquáticos, em especial, daqueles constantemente usados no fornecimento de água potável ou ainda localizados em grandes centros urbanos, pois altas taxas de produção primária de macrófitas aquáticas ou de fitoplâncton podem causar mau cheiro, auxiliar na proliferação de mosquitos, como ocorreu na hidrelétrica de Tucuruí (Ribeiro et al. 1995), ou mesmo comprometer atividades de lazer entre outros usos.

Durante um longo tempo a eutrofização foi a principal característica do Lago Paranoá em Brasília. Formado em 1959, um ano antes da inauguração da nova capital do Brasil, o reservatório desde então passou a receber os esgotos domésticos da cidade, além de outras entradas difusas de fósforo, e de despejos de matadouros clandestinos.

Esta situação levou, no final da década de 70, ao que é considerado o maior desastre ecológico do lago: um "bloom" da cianobactéria Microcystis aeruginosa, concentrada principalmente num dos braços do reservatório (Riacho Fundo), causando a primeira grande mortandade de peixes, que se repetiram outras vezes na década de 80 (Burnett 2001).

Devido à esta conjuntura, a CAESB (Companhia de Abastecimento e Saneamento de Brasília) iniciou em 1976 um programa de monitoramento, que consistia em analisar a água nos quatro grandes braços do
Lago Paranoá e no seu corpo central, com o objetivo de tentar manejar o lago e conter a diminuição da qualidade de água do reservatório.

Mas os problemas continuaram e, em 1982, foi necessário fazer a retirada manual e com dragas da macrófita Eichhornia crassipes. Nesta época a percepção pública dos problemas do lago aumentou e a imprensa noticiou: "Brasília fede" (Starling \& Lazzaro 1997). Em 1997, cerca de 150 toneladas de tilápias foram recolhidas após nova mortandade no braço Riacho Fundo (Starling et al. 2002a).

Como alternativa, a CAESB implantou duas novas Estações de Tratamento de Esgoto (ETE), a ETE Norte e a ETE Sul, que entraram em operação em 1993, com um avançado sistema de tratamento terciário que reduziu em $70 \%$ o aporte de fósforo e nitrogênio ao lago (Cavalcanti et al. 1997).

Neste trabalho o conjunto de dados do monitoramento de longa duração da CAESB ( 25 anos), foi usado para avaliar os efeitos de três intervenções de manejo (instalação das ETE; instalação efetiva e flushing) sobre a dinâmica temporal das variáveis Clorofila-a, Fósforo Total e Transparência medidas em diferentes pontos do Lago Paranoá entre 1976 e 2001.

\section{MATERIAL E MÉTODOS}

O reservatório do Lago Paranoá tem $38 \mathrm{~km}^{2}$, com profundidade média de $14 \mathrm{~m}$, tempo de residência da água de 0.82 anos e volume de $498^{*} 10^{6} \mathrm{~m}^{3}$ e está localizado dentro do perímetro urbano da cidade de Brasília (Starling et al. 2002a). Possui quatro braços formados por diferentes riachos: Riacho Fundo (ponto de amostragem A), Gama (ponto B), Bananal (ponto E) e Torto (D). O ponto $\mathrm{C}$ está localizado no corpo central do reservatório (Figura 1).

Os dados analisados neste trabalho foram obtidos entre 09/09/1976 e 23/05/2001, e as medidas foram semanais no início, passando a quinzenais em 1988 e à partir de 1997, mensais. As concentrações de Fósforo Total $\left(\mu \mathrm{g}^{*} \mathrm{~L}^{-1}\right)$ e Clorofila-a $\left(\mu \mathrm{g}^{*} \mathrm{~L}^{-1}\right)$ foram medidas na superfície e determinadas seguindo os métodos padronizados APHA (1998). A transparência (cm) foi aferida utilizando-se disco de Secchi.

Para as análises foi usada apenas uma medida para cada mês. Nos meses com mais de uma medida, foi sorteada apenas uma das medições para compor a planilha final da análise. 


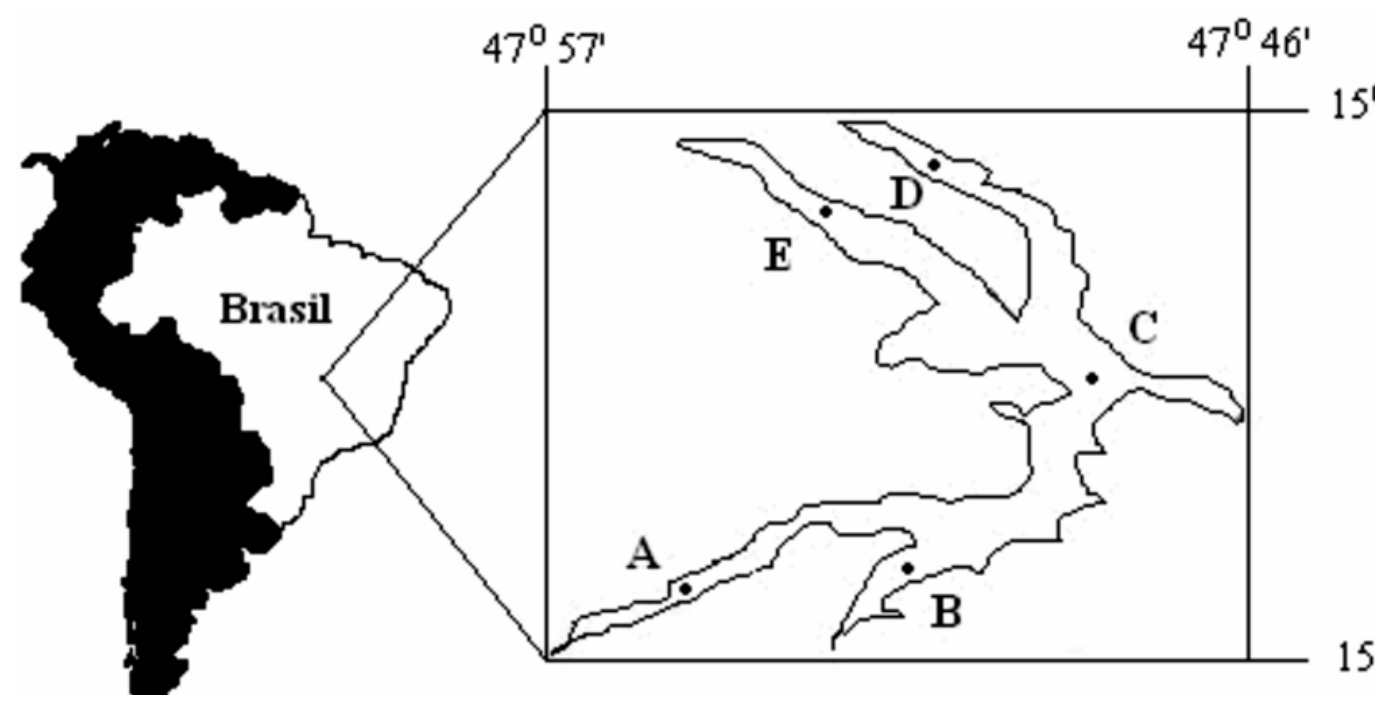

Figura 1. Lago Paranoá (DF), com pontos de coleta nos respectivos braços: Riacho Fundo (A), Gama (B), Bananal (E), Torto (D) e corpo central (C). Figure 1. Geographical location of Paranoá lake, displaying the sampling sites over the extensions: Riacho Fundo (A), Gama (B), Bananal (E), Torto (D) and main portion $(C)$.

\section{ANÁLISE ESTATÍSTICA}

A análise de intervenção em séries temporais (Box \& Tiao 1975), que tem por objetivo avaliar o impacto de uma intervenção no comportamento de uma série, foi usada para comparar as três variáveis nos cinco pontos de coleta de dados no período de 25 anos. A técnica da análise de intervenção é a abordagem mais adequada para determinar se mudanças significativas ocorreram após a intervenção (Morettin \& Toloi 2004).

Considerando a variável de interesse como $\mathrm{Y}$, o modelo geral para uma análise de intervenção é:

$$
\mathrm{Y}_{\mathrm{t}}=p \mathrm{Y}_{\mathrm{t}-1}+\varpi \mathrm{I}_{\mathrm{t}}+e_{\mathrm{t}}
$$

Onde:

$I_{t}=0$ antes da intervenção e $I_{t}=1$ depois da intervenção, ఐ é o coeficiente que mede a "função degrau" ("step function"), isto é, mede a diferença no nível médio da série antes e depois da intervenção, por isto possui a mesma unidade de medida da variável em questão, $p$ é o coeficiente auto-regressivo e $e_{\mathrm{t}}$ é o erro aleatório num dado tempo $t$.

Para confirmar a adequação do modelo, foi calculado o coeficiente de correlação serial dos resíduos, que mede a correlação da variável com ela mesma com a defasagem temporal de um mês. Valores baixos para este coeficiente significam que o modelo é apropriado para avaliar as intervenções (Manly 1994).

Três intervenções foram analisadas neste trabalho: $1^{\text {a }}$ - o início das operações das ETE (01/1993); $2^{\mathrm{a}}$ - o pleno funcionamento destas ETE que dada a complexidade de seus procedimentos e falta de pessoal devidamente treinado, começou atuar em sua plenitude apenas em 01/1996; e, por último, a $3^{\mathrm{a}}$ - um evento de flushing que consistiu na abertura abrupta das comportas do reservatório, eliminando principalmente a água superficial (11/1999).

Assim, o modelo de intervenção acima foi ajustado com três coeficientes $(\varpi 1, \varpi 2, \varpi 3)$, cada um representando uma das intervenções. O modelo foi calculado para cada um dos cinco pontos e para cada uma das três variáveis, totalizando, então 15 modelos, cada um com três coeficientes medindo as respectivas intervenções.

\section{RESULTADOS}

Fósforo Total e Clorofila-a foram as variáveis que tiveram uma dinâmica bastante irregular até meados de 1995, quando seus valores começaram a diminuir e as amplitudes das oscilações a declinar, apesar disto a transparência só passou a ser alta em 1999-2000. Este padrão pode ser visto em todos os cinco pontos do lago como mostram as Figuras 2 (pontos A e B), 3 (pontos D e E) e 4 (ponto $\mathrm{C}$ ou corpo central do reservatório). 

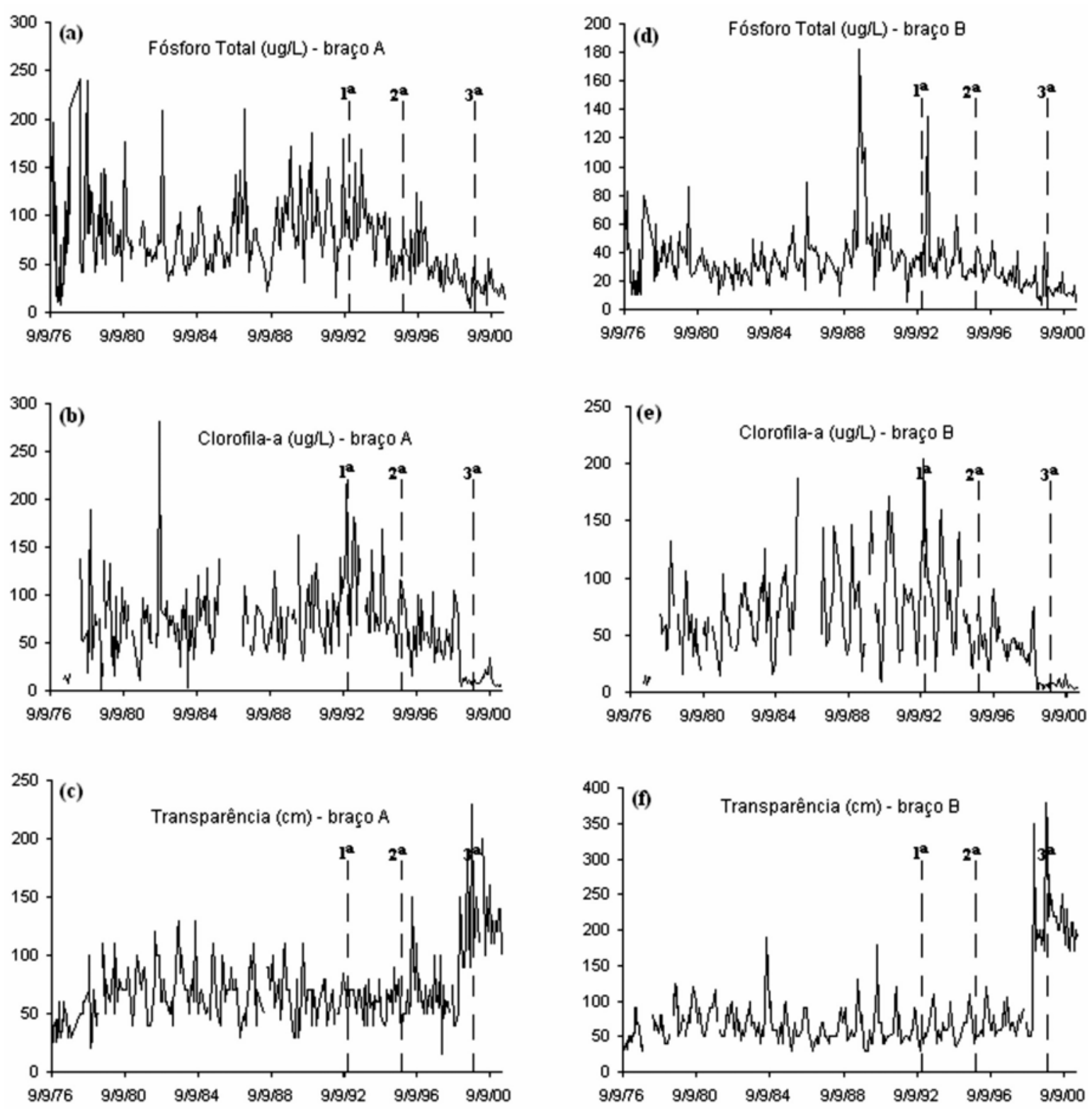

Figura 2. Variação de Fósforo Total $\left(\mu \mathrm{g}^{*} \mathrm{~L}^{-1}\right)$, Clorofila-a $\left(\mu \mathrm{g}^{*} \mathrm{~L}^{-1}\right)$ e Transparência (cm) para os braços do Riacho Fundo (A) e do Gama (B) no Lago Paranoá. $1^{\mathrm{a}}$ intervenção: 01/1993; $2^{\mathrm{a}}$ intervenção: 01/1996; $3^{\mathrm{a}}$ intervenção: 11/1999.

Figure 2. Temporal variation at sampling sites Riacho Fundo (A) and Gama (B) of Paranoá lake of the variables Total Phosphorous ( $\left.\mu g^{*} L^{-1}\right)$, Chlorophyll-a $\left(\mu \mathrm{g}^{*} \mathrm{~L}^{-1}\right)$ and Transparency $(\mathrm{cm})$. The moment of the three human interventions are indicated with dotted vertical lines at dates $01 / 1993$, 01/1996, and 11/1999.

Os resultados dos modelos, incluindo os coeficientes (ఐ) das intervenções, nos cinco pontos amostrados, são descritos para cada uma das variáveis na Tabela I.

Todos os 15 modelos da Tabela I são significativos e apresentaram baixos coeficientes de correlação, isto é, são adequados para descrever o fenômeno proposto. Exceções poderiam ser os modelos para Clorofila-a dos pontos $\mathrm{B}$ e $\mathrm{C}$ com valores de correlação serial 0,14 e 0,16 respectivamente. Contudo, estes valores são ainda baixos e os altos valores dos coeficientes de intervenção (cuja unidadeé $\mu \mathrm{g}^{*} \mathrm{~L}^{-1}$ ) especialmentepara o ponto $\mathrm{B}$, mostram que a diminuição da Clorofila-a foi bastante acentuada, apesar de estatisticamente não significativa (veja os valores de $\varpi 1$ e $\varpi 3$ para o ponto $\mathrm{B}$ e o valor de $\varpi 3$ para o ponto $\mathrm{C}$ ).

Nota-se pela Tabela I que apesar de todas as três intervenções colaborarem para a diminuição da Clorofila-a (valores de $\varpi$ são negativos), todos os valores de ๗1 não são significativos, isto é, para a Clorofila-a apenas à partir da segunda intervenção (funcionamento adequado das ETE), a Clorofila-a foi reduzida.

Os valores de Fósforo Total também foram reduzidos, apenas à partir da segunda intervenção, mas principalmente com a terceira (flushing), quando 

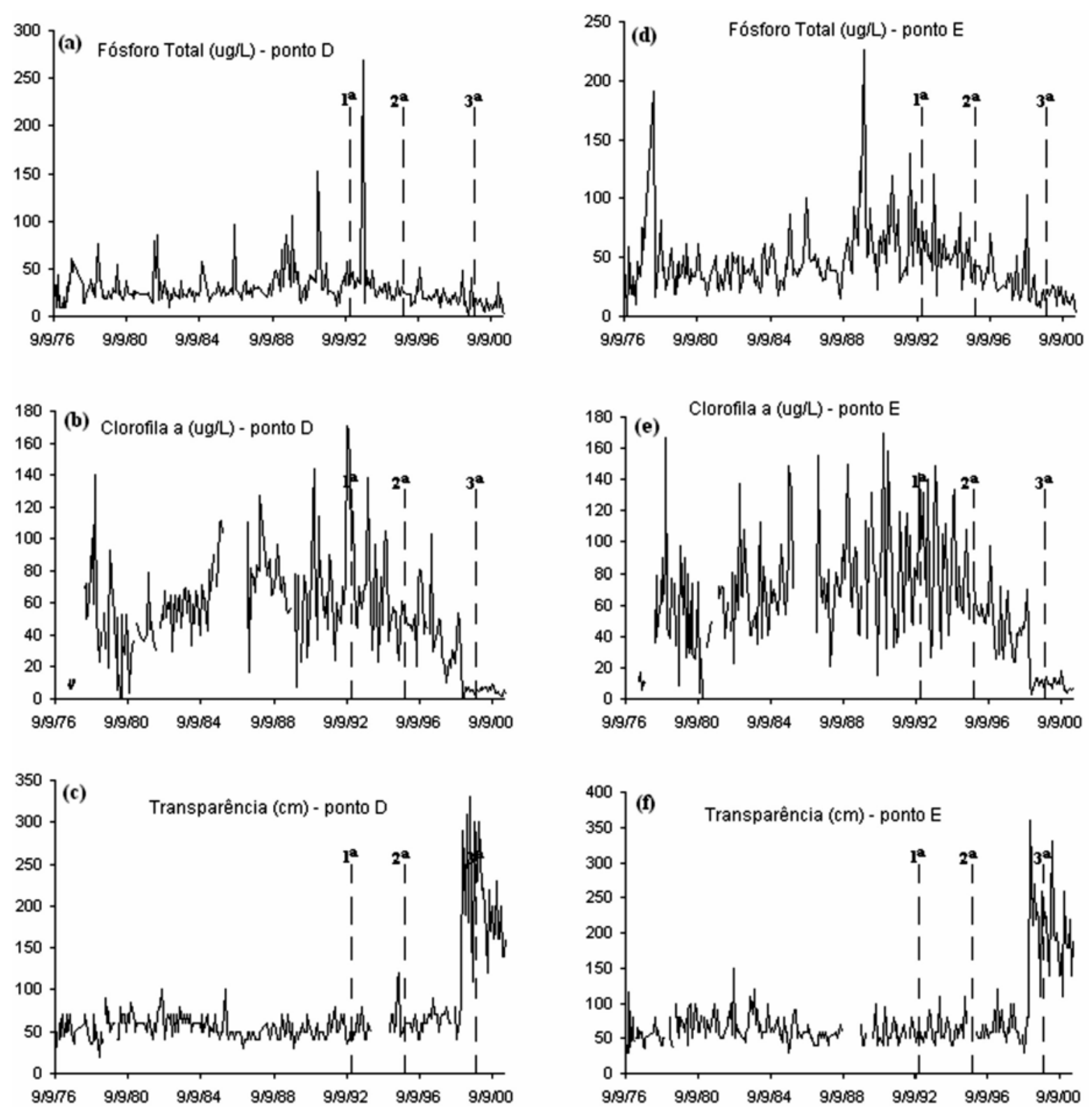

Figura 3. Variação de Fósforo Total $\left(\mu \mathrm{g}^{*} \mathrm{~L}^{-1}\right)$, Clorofila-a $\left(\mu \mathrm{g}^{*} \mathrm{~L}^{-1}\right)$ e Transparência $(\mathrm{cm})$ para os braços do Bananal (E) e do Torto (D) no Lago Paranoá. $1^{\mathrm{a}}$ intervenção: 01/1993; $2^{\mathrm{a}}$ intervenção: 01/1996; $3^{\mathrm{a}}$ intervenção: 11/1999.

Figure 3. Temporal variation at sampling sites Bananal (E) and Torto (D) of Paranoá lake of the variables Total Phosphorous $\left(\mu g^{*} L^{-1}\right)$, Chlorophyll-a $\left(\mu g^{*} L^{-1}\right)$ and Transparency (cm). The moment of the three human interventions are indicated with dotted vertical lines at dates 01/1993, 01/1996, and 11/1999.

todos os valores $\varpi 3$ são altamente significativos e em torno de $0,65 \mu \mathrm{g} * \mathrm{~L}^{-1}$.

A transparência diferencia-se das outras variáveis, pois apenas a terceira intervenção foi estatisticamente significativa, com aumentos de $0,5 \mathrm{~m}$ no ponto A e de mais de $1,2 \mathrm{~m}$ nos outros pontos, chegando mesmo a quase $2 \mathrm{~m}$ no corpo central do reservatório (ponto $\mathrm{C}$ ).

\section{DISCUSSÃO}

Quando a cidade de Brasília foi inaugurada em 1960, a população total da nova capital federal foi estimada para alcançar no máximo 500 mil habitantes nos próximos 50 anos, mas hoje já ultrapassa 2,4 milhões, com pelo menos $50 \%$ dela vivendo na bacia do Paranoá (Fonseca 2001). O lago Paranoá havia sido construído com o intuito principal de aumentar a umidade da cidade e produzir eletricidade, mas desde sempre foi usado para atividades de lazer, pesca e para despejo de efluentes (França et al. 1964).

Assim, a população crescente aliada à falta de tratamento de esgotos domésticos, levou à eutrofização do lago Paranoá, com alta quantia de algas e macrófitas aquáticas e que, às vezes, exalava um grande mau 
cheiro. Starling et al. (2002a) argumentam que este mau cheiro era provocado por mudanças abruptas de temperatura que aumentavam a circulação da água e re-suspendiam o sedimento causando anoxia em toda a coluna d'água e a mortalidade de muitos organismos, incluindo peixes.
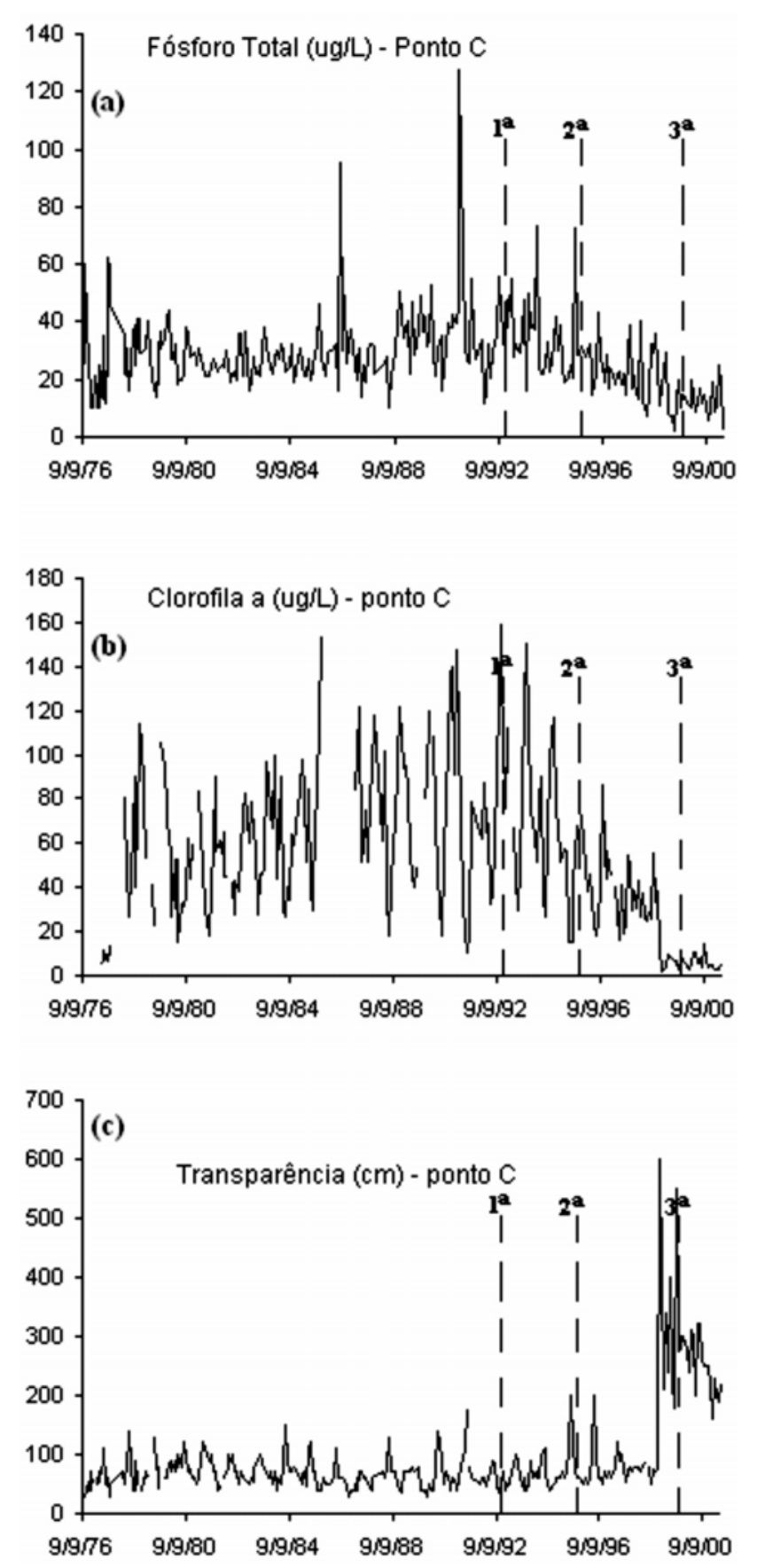

Figura 4. Variação de Fósforo Total $\left(\mu g^{*} L^{-1}\right)$, Clorofila-a $\left(\mu g^{*} L^{-1}\right)$ e Transparência $(\mathrm{cm})$ para o corpo central (ponto C) do Lago Paranoá. $1^{\mathrm{a}}$ intervenção: 01/1993; $2^{\mathrm{a}}$ intervenção: 01/1996; $3^{\mathrm{a}}$ intervenção: 11/1999. Figure 4. Temporal variation at the sampling site on the main portion of Paranoá lake of the variables Total Phosphorous $\left(\mu \mathrm{g}^{*} \mathrm{~L}^{-1}\right)$, Chlorophyll-a $\left(\mu{ }^{*} L^{-1}\right)$ and Transparency (cm). The moment of the three human interventions are indicated with dotted vertical lines at dates 01/1993, 01/1996; and 11/1999.
A influência de outras variáveis nestes eventos, mostra que a Clorofila-a não depende apenas do fósforo, que aqui não foram analisadas. Por causa disto, a relação entre fósforo e clorofila-a não é tão clara para este mesmo conjunto de dados como demonstraram Starling et al. (2002b) e outras variáveis devem ter influenciado as altas oscilações de Clorofila-a em todo o reservatório (Figuras 2, 3 e 4).

Devido a estas oscilações e riscos imprevistos de mau cheiro, a CAESB implantou um sistema de monitoramento que, posteriormente, fundamentou a instalação das Estações de Tratamento de Esgoto, mostrando que o monitoramento pode ter resultados práticos para a adoção de medidas de manejo, mesmo que não muito baratas, como neste caso.

Por outro lado, foi este mesmo monitoramento que avaliou que as ETE não estavam sendo eficientes, como comprovam os resultados da primeira intervenção ( 1 , a inauguração das ETE), para as três variáveis aqui analisadas e que não detectaram diferenças significativas, antes e depois da intervenção (Tabela I). Desta maneira, os resultados do monitoramento evidenciaram a necessidade de treinamento de pessoal que foi realizado e, então, quando as ETE passaram a operar adequadamente (início de 1996), ocorreu a diminuição, estatisticamente significativa, dos valores das variáveis Clorofila-a e Fósforo Total (segunda intervenção - ఐ2). Vale ressaltar, que as análises aqui realizadas são inéditas e que a CAESB chegou a estas conclusões, em parte, observando os gráficos das séries temporais do monitoramento.

Apesar disto, a variável mais perceptível pelos usuários de um reservatório urbano é a transparência da água e esta não sofreu diferença significativa, na segunda intervenção (Tabela I), pois a visibilidade aumentou em média apenas nove centímetros nos cinco pontos amostrados.

Desta forma foi necessário um evento conhecido como flushing, que abriu abruptamente as comportas do reservatório retirando a camada superficial da água e consequentemente todo o plâncton contido nesta zona eufótica, para que a transparência do lago Paranoá, aumentasse significativamente (Tabela I, ఐ3) em mais de um metro para a maioria dos pontos.

A hipótese mais provável para explicar este fenômeno, é que apesar da diminuição anterior de $70 \%$ do aporte de fósforo ao lago (Cavalcanti et al. 1997) e também da quantia de Fósforo Total e Clorofila-a, já à 
Tabela I. Resultados da Análise de Intervenção aplicada aos dados de Clorofila-a $\left(\mu \mathrm{g}^{*} \mathrm{~L}^{-1}\right)$, Fósforo Total $\left(\mu \mathrm{g}^{*} \mathrm{~L}^{-1}\right)$ e Transparência $(\mathrm{cm})$ para cada um dos pontos de amostragem (A, B, C, D, e E) localizados no Lago Paranoá em Brasília. $p(1)$ : parâmetro autoregressivo de ordem 1 (todos os modelos são significativos); ๗1: coeficiente da "função degrau" (aumento ou diminuição, veja sinal) para a primeira intervenção e nível de significância associado entre parênteses; ๗2: coeficiente para a segunda intervenção e ๘3: coeficiente para a terceira; $r$ serial: coeficiente de autocorrelação dos resíduos (valores baixos indicam boa adequação do modelo).

Table I. Resultados da Análise de Intervenção aplicada aos dados de Clorofila-a ( $\left.\mu \mathrm{g}^{*} \mathrm{~L}^{-1}\right)$, Fósforo Total $\left(\mu \mathrm{g}^{*} L^{-1}\right)$ e Transparência (cm) para cada um dos pontos de amostragem ( $A, B, C, D$, e E) localizados no Lago Paranoá em Brasília. p(1): parâmetro autoregressivo de ordem 1 (todos os modelos são significativos); $\varpi 1$ : coeficiente da "função degrau" (aumento ou diminuição, veja sinal) para a primeira intervenção e nivel de significância associado entre parênteses; $\varpi 2$ : coeficiente para a segunda intervenção e $\varpi 3$ : coeficiente para a terceira; $r$ serial: coeficiente de autocorrelação dos resíduos (valores baixos indicam boa adequação do modelo).

\begin{tabular}{lllllll}
\hline Variável & Ponto & $\mathbf{p}(\mathbf{1})$ & $\varpi \mathbf{1}$ & $\varpi \mathbf{2}$ & $\varpi \mathbf{3}$ & r serial \\
\hline Clorofila-a & $\mathrm{A}$ & 0,37 & $12,9(0,160)$ & $-36,7(0,000)$ & $-35,8(0,001)$ & 0,02 \\
\hline Clorofila-a & $\mathrm{B}$ & 0,64 & $-12,6(0,325)$ & $-34,3(0,017)$ & $-15,3(0,331)$ & 0,14 \\
\hline Clorofila-a & $\mathrm{C}$ & 0,63 & $-2,0(0,843)$ & $-32,5(0,005)$ & $-31,0(0,010)$ & 0,16 \\
\hline Clorofila-a & $\mathrm{D}$ & 0,44 & $-9,3(0,253)$ & $-22,8(0,016)$ & $-30,2(0,002)$ & 0,00 \\
\hline Clorofila-a & $\mathrm{E}$ & 0,27 & $-0,18(0,98)$ & $-34,0(0,000)$ & $-34,1(0,000)$ & 0,04 \\
\hline Fósforo Total & $\mathrm{A}$ & 0,46 & $-0,03(0,79)$ & $-0,43(0,016)$ & $-0,68(0,000)$ & $-0,06$ \\
\hline Fósforo Total & $\mathrm{B}$ & 0,45 & $0,00(0,99)$ & $-0,37(0,17)$ & $-0,60(0,000)$ & $-0,06$ \\
\hline Fósforo Total & $\mathrm{C}$ & 0,35 & $0,106(0,31)$ & $-0,39(0,004)$ & $-0,67(0,000)$ & 0,00 \\
\hline Fósforo Total & $\mathrm{D}$ & 0,36 & $0,106(0,39)$ & $-0,44(0,007)$ & $-0,56(0,000)$ & $-0,03$ \\
\hline Fósforo Total & $\mathrm{E}$ & 0,48 & $0,128(0,35)$ & $-0,40(0,027)$ & $-0,89(0,000)$ & $-0,05$ \\
\hline Transparência & $\mathrm{A}$ & 0,27 & $-6,96(0,217)$ & $7,27(0,324)$ & $55,0(0,000)$ & $-0,01$ \\
\hline Transparência & $\mathrm{B}$ & 0,39 & $-2,89(0,739)$ & $11,45(0,312)$ & $124,2(0,000)$ & 0,00 \\
\hline Transparência & $\mathrm{C}$ & 0,16 & $1,92(0,841)$ & $8,03(0,525)$ & $181,1(0,000)$ & 0,00 \\
\hline Transparência & $\mathrm{D}$ & 0,30 & $1,39(0,831)$ & $8,47(0,325)$ & $126,1(0,000)$ & $-0,01$ \\
\hline Transparência & $\mathrm{E}$ & 0,19 & $0,26(0,961)$ & $1,79(0,822)$ & $129,5(0,000)$ & $-0,01$ \\
\hline
\end{tabular}

partir da segunda intervenção (Tabela I), a abundância de fitoplâncton e do fósforo restante no lago era suficiente para se retro-alimentar, ou seja, a mortalidade das algas disponibilizava fósforo ao lago, que por sua vez era reabsorvido novamente para o aumento da produtividade primária. O flushing terminou com este ciclo fazendo com que o Lago tenha nos dias atuais áreas com transparência (visibilidade) da ordem 6 metros, inclusive recebendo praticantes de pesca subaquática para a captura de carpas com arpão (F. Starling, observação pessoal).

Bicudo et al. (2007) avaliaram, utilizando-se também da análise de intervenção, a retirada de macrófitas aquáticas do reservatório de Garças (SP) e caracterizaram esta intervenção como um abrupto impacto permanente no ecossistema local. Pode-se afirmar que o Lago Paranoá também atingiu, com um abrupto impacto (flushing), um novo e permanente nível de produtividade primária e consequentemente equilíbrio.

Este novo patamar de produção primária, também reduziu o número de pescadores artesanais do lago, já que o principal produto da pesca era a tilápia Oerochromis niloticus, que chegou a sustentar ao menos 100 famílias de pescadores na década de 80 e hoje emprega somente 53 (Walter \& Petrere 2007). Apesar disto, esta pescaria tem um rendimento superior ao da represa Billings em São Paulo, com produtividade primária muito maior (Petrere et al. 2006).

\section{CONCLUSÃO}

As críticas mais comuns aos monitoramentos são que eles não fazem parte da "ciência real", que dados de monitoramento nunca são usados e que não é possível saber hoje, quais as questões que serão feitas no futuro (Lovett et al. 2007). Apesar destas considerações, não há outra maneira de se entender as mudanças de longo prazo nos ecossistemas se dados e informações de monitoramento não existirem ou mesmo se não forem disponibilizados.

Através de dados de monitoramento da CAESB, este trabalho mostrou que o controle do fósforo é condição essencial para a diminuição da produtividade primária, mas não suficiente, pois foi necessária uma terceira intervenção, oflushing, para que o efeito de feedback na zona eufótica cessasse e reduzisse os valores de Clorofila-a e Fósforo Total. 
O aumento da transparência de um lago urbanizado amplia a percepção pública de que o tratamento do esgoto resulta em benefícios reais para o ambiente e para a sociedade. Isto influencia as decisões dos órgãos envolvidos, entre elas o monitoramento ambiental que é fundamental para a compreensão dos sistemas e indispensável para a formulação e a implantação de medidas de manejo.

\section{REFERÊNCIAS}

APHA 1998. Standard Methods for the Examination of Water and Wastewater, 20th Ed. APHA (American Public Health Association), Washington, DC. 567p.

ARIAS,A.R.L.; BUSS, D.F.;ALBUQUERQUE, C.; INÁCIO,A.F.; FREIRE, M.M.; EGLER, M.; MUGNAI, R. \& BAPTISTA, D.F. 2007. Utilização de bioindicadores na avaliação de impacto e no monitoramento da contaminação de rios e córregos por agrotóxicos. Ciência \& Saúde Coletiva, 12(1): 61-72.

BICUDO, D.C.; FONSECA, B.M.; BINI, L.M.; CROSSETI, L.O.; BICUDO, C.M. \& ARAÚJO-JESUS, T. 2007. Undesirable side-effects of water hyacinth control in a shallow tropical reservoir. Freshwater Biology, 52: 1120-1133.

BOX, G.E. \& TIAO, G.C. 1975. Intervention analysis with applications to economic and environmental problems. Journal of the American Statistics Association, 70, 70-79.

BURNETT, J.A.B.; MATTOS, S.P. \& AZZOLIN, N.M.P. 2001. Intervenções da Companhia de Saneamento. pp. 199-212. In: F.O. Fonseca, (org.). Olhares sobre o Lago Paranoá. Ed. SEMARH Brasília. 420p.

CAVALCANTI, C.G.B.; PINTO, M.T.; FREITAS, H.J. \& MOREIRA, R.C.A. 1997. Paranoá Lake restoration: impact of tertiary treatment sewage in the watershed. Verhandlungen der Inernationalen Vereinigun für Theoretische und Angewandte Limnologie, 26: 689-693.

FRANÇA, V.C.; GURGEL, J.J.S. \& BRAGA, R.A. 1964. Exame biológico-pesqueiro do Lago "Israel Pinheiro", Brasília, Brasil. Relatório apresentado ao Diretor da então Diretoria de Fomento e Produção da DNOCS, p. 35-53.

FONSECA, F.O. (org.). 2001. Olhares sobre o Lago Paranoá. Ed. SEMARH Brasília 420p.

LOVETT, G.M.; BURNS, D.A.; DRISCOLI, C.T.; JENKINS, J.C.; MITCHELL, M.J.; RUSTAD, L.; SHANLEY, J.B.; LIKENS, G.E. \& HAEUBER, R. 2007. Who needs environmental monitoring? Frontiers in Ecology and the Environment, 5(5): 253-260.

MANLY, B.F.J. 1994. The Design and Analysis of Research Studies. Cambridge University Press, Cambridge. 376p.
MATTHEWS, R. A.; BUIKEMA, A. L. \& CAIRNS Jr., J. 1982. Biological monitoring part IIA: Receiving system functional methods relationships, and indices. Water Research, 16: 129-139.

MILESSI, A.C.; ARANCIBIA, H.; NEIRA, S. \& DEFEO, O. 2005. The mean trophic level of Uruguayan landings during the period 1990-2001. Fisheries Research, 74: 223-231.

MORETTIN, P.A. \& TOLOI, C.M. 2004. Análise de Séries Temporais. Edgar Blucher Editora. 535p.

PETRERE Jr., M.; WALTER, T. \& MINTE-VERA, C.V. 2006. Income evaluation of small-scale fishers in two Brazilian urban reservoirs: repress Billings (SP) and Lago Paranoá (DF). Brazilian Journal of Biology, 66(3): 817-828.

RIBEIRO, M.C.L.B.; PETRERE-JR. M. \& JURAS, A.A. 1995. Ecological integrity and fisheries ecology of the AraguaiaTocantins River Basin, Brazil. Regulated Rivers: Research \& Management, 11: 325-350.

SHIGAKI, F.; SHARPLEY, A. \& PROCHNOW, L.I. 2006. Animal-based agriculture phophorus management and water quality in Brazil: options for the future. Scientia Agricola, 63(3): 194-209.

STARLING, F.L.R.M. \& LAZZARO, X. 1997. Experimental investigation of feasilility of improving water quality by controlling exotic planktivore overpopulation in the eutrophic Paranoá Reservoir no. Brasilia, Brazil. Verh. Internat. Verein. Limnol., 26: 789-794

STARLING, F.L.R.M.; LAZZARO, X.; CAVALCANTI, C. \& MOREIRA, R. 2002a. Contribution of omnivorous tilapia to eutrophication of a shallow tropical reservoir: evidence from a fish kill. Freshwater Biology, 47: 2443-2452.

STARLING, F.L.R.M.; PEREIRA, C.E.B. \& ANGELINI, R. 2002b. Definição da capacidade suporte do Lago Paranoá, para a ocupação urbana de sua bacia de drenagem. Relatório técnico apresentado a CEB - Companhia Energética de Brasília (Brasília - DF). 340p.

WATER, T. \& PETRERE Jr., M. 2007. The small scale urban reservoir fisheries of Lago Paranoá, Brasília, DF, Brasil. Brazilian Journal of Biology, 67(1): 9-21.

Submetido em 16/02/2008. Aceito em 22/07/2008. 